Institute of $\mathbf{F}_{\text {ood and }} \mathbf{A}_{\text {gricultural }} \mathbf{S}_{\text {ciences }}$

\title{
Nematode (Roundworm) Infections in Fish ${ }^{1}$
}

Roy P.E. Yanong ${ }^{2}$

\section{Introduction}

Nematodes, or roundworms, infect many different species of aquacultured and wild fish. Small numbers of nematodes often occur in healthy fish, but high numbers will cause illness or even death. In aquaculture systems, brood stock infected with a small number of nematodes may not even show signs of illness, but often have reduced productivity rates. On the other hand, juvenile fish infected by small numbers of nematodes are more likely to show signs of illness and also have reduced growth rates.

In aquaculture situations, fish become infected with nematodes if they are fed live foods containing infective life stages or if they are raised in culture settings that promote the growth of other animals that allow nematodes to complete their life cycle (intermediate hosts) or carry the infective stages of the nematode (vector or paratenic host). Finally, some nematodes can be transmitted directly from fish to fish.

Adult nematodes are typically found in fish digestive tracts. However, depending upon the species of nematode and the species of infected fish, adult and other life stages of nematodes can be found in almost any part of the fish, including the internal organs, the swim bladder, deeper layers of the skin or fins, and external muscle layers.

Prevention, proper identification, and correct therapy for treatable infestations dramatically improve the health and productivity of affected fish.

\section{Identification}

Identification is best done with a microscope. Nematodes are smooth, cylindrical, relatively long worms (Figure 1), which distinguishes them from the flatter, segmented tapeworms (Figure 2) and from the stouter and shorter monogenes (flukes) (Figure 3). Pentastomids (Figure 4) are another group of parasites that can be confused with nematodes, but they are considerably more stout and have more visible segmentation.

Acanthocephalans (Figure 5) are also somewhat similar in appearance, but they have a characteristic "thorny head" - their head region is armed and encircled with numerous rows of hooks.

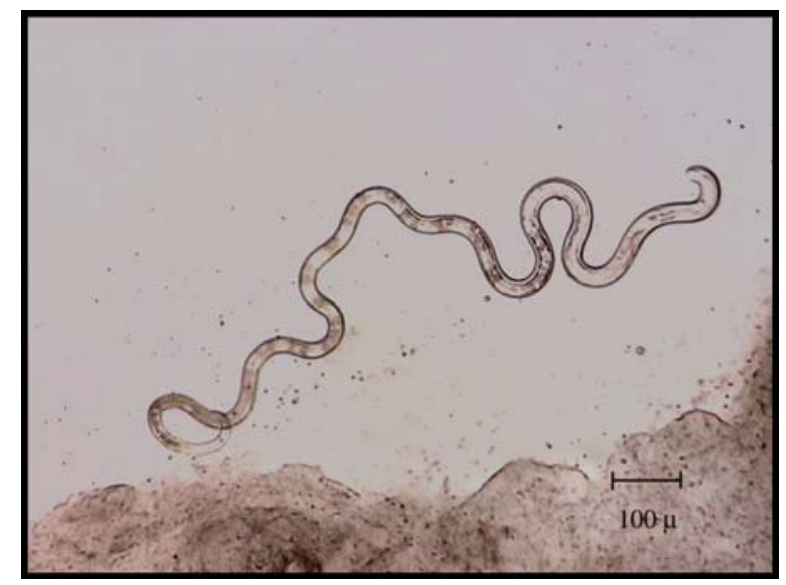

Figure 1. Photomicrograph of a Capillaria nematode showing typical nematode shape.

\footnotetext{
${ }^{1}$ This document is Circular 91, one of a series from the Department of Fisheries and Aquatic Sciences, Florida Cooperative Extension Service, Institute of Food and Agricultural Sciences, University of Florida. First published: December 2002.

${ }^{2}$ Roy P.E. Yanong, Assistant Professor, Tropical Aquaculture Laboratory, Department of Fisheries and Aquatic Sciences, Florida Cooperative Extension Service, Institute of Food and Agricultural Sciences, University of Florida, Ruskin, FL 33570-3434. 


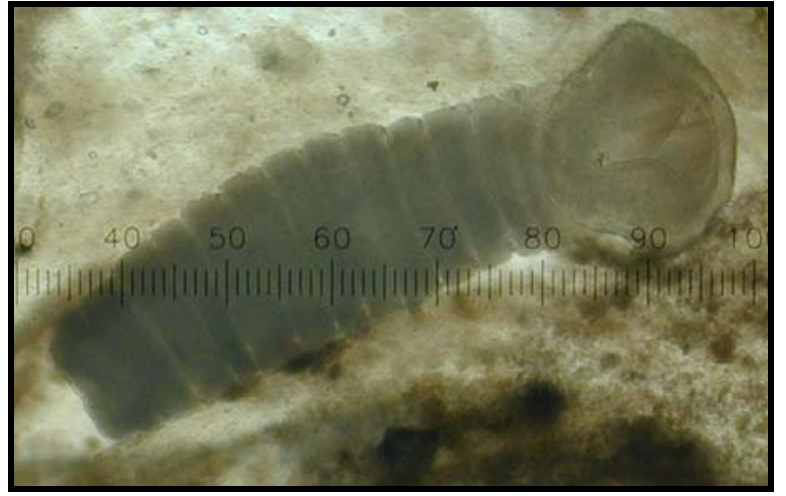

Figure 2. Photomicrograph of a tapeworm showing typical segmentation (scale in micrometers - tapeworms can be much longer than the one shown).

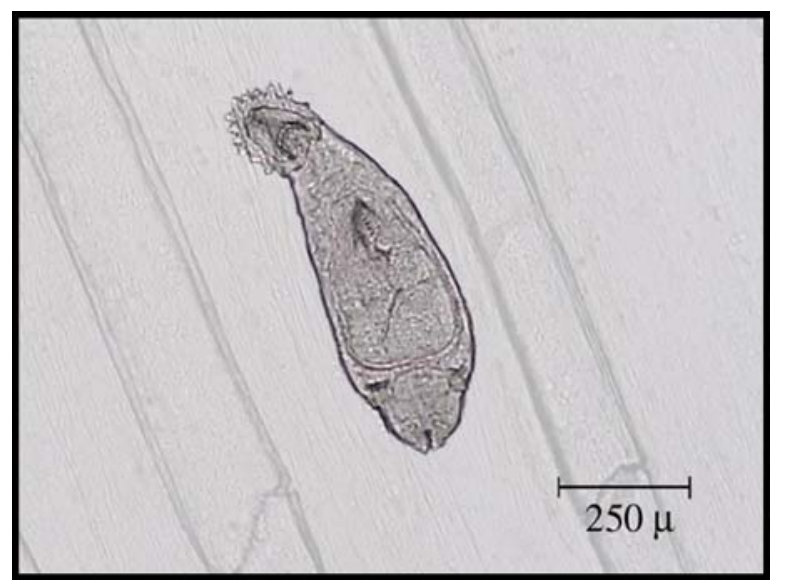

Figure 3. Photomicrograph of a monogene (fluke) showing typical shape.

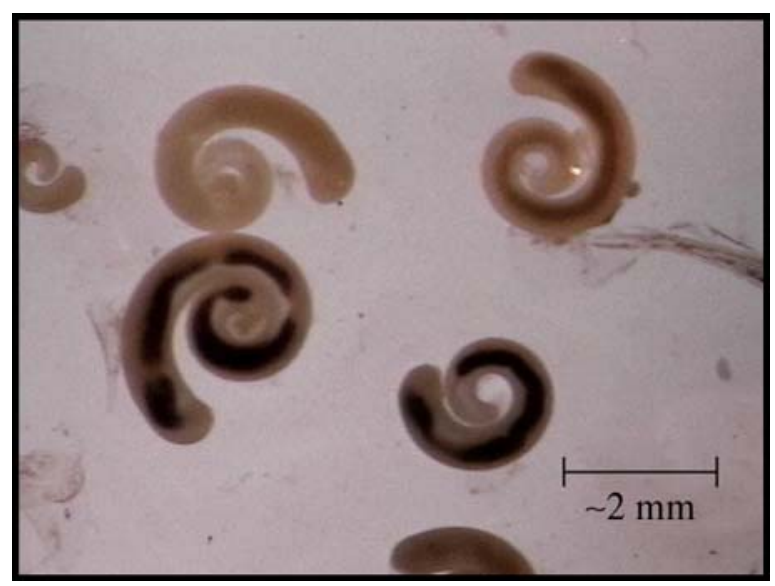

Figure 4. Photomicrograph of pentastomes showing typical shape.

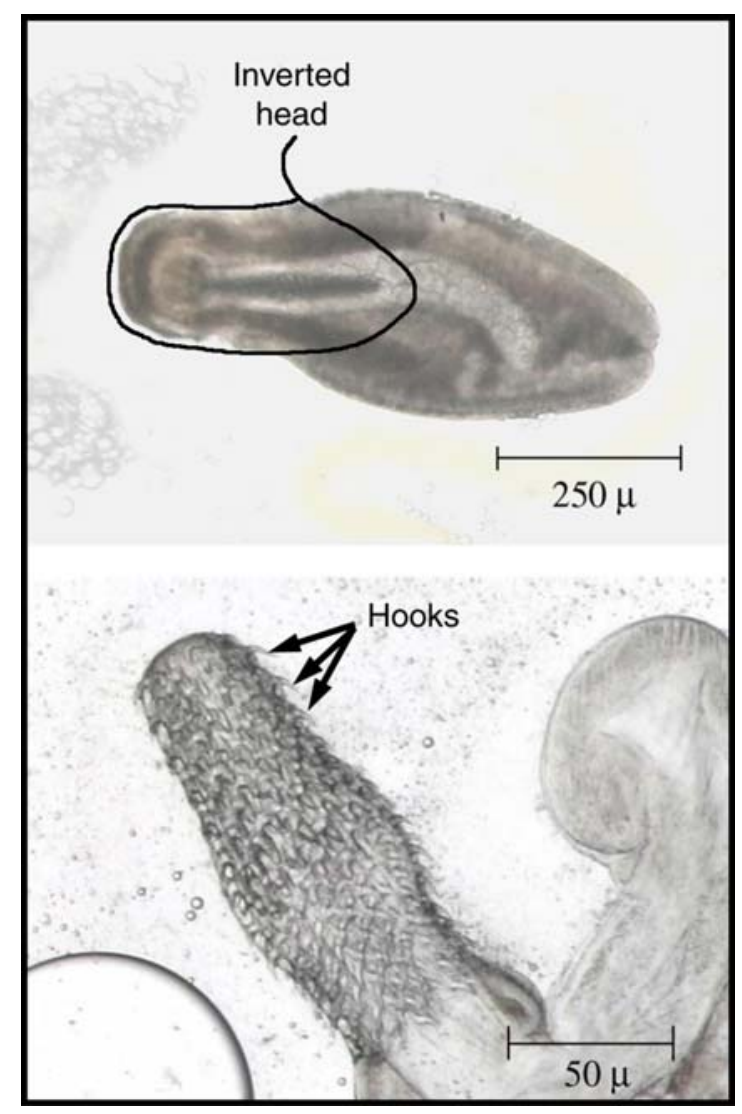

Figure 5. Photomicrograph showing acanthocephalan with head inverted (top) and head everted showing typical "thorny head" with hooks (bottom).

\section{Disease in Fish}

The severity of disease in fish will vary depending upon the life stage, species, and number of nematodes present; the age and species of infected fish; and the sites of infection. Even though adult nematodes are typically found in fish intestinal tracts, adult and other life stages can be found in almost any organ, as stated earlier, but they are most commonly present in muscle, the liver, and tissues surrounding the internal organs. Visible signs of infection may include hemorrhaging, cysts or granuloma formation (a granuloma is a reaction by immune cells in which the cells try to "wall off" some foreign body - in this case, the worm. Granulomas formed around worms can look like little brown "rock-like" areas in the shape of the worm, but will be surrounded by a distinct clear area at their very edge), external lumps or nodules, inflammation, and necrosis (presence of dead and dying tissue). Adult nematodes in the intestinal tract damage its lining and rob the fish of nutrients, causing a "wasting" effect. 
During their development, many species of nematodes migrate within the body of a fish causing "worm tracks" (evidence of nematode movement through a fish's body), which are seen as "tunnels" in the tissue. Inflammation around these "worm tracks" may also be present. Extensive migration by large numbers of nematodes may cause significant physical damage to a fish. Large larval stages of some nematode species, including Eustrongylides species, may cause physical pressure on organs and abdominal distension.

Juvenile fish with nematode infections are often more severely affected than adults, displaying reduced growth, wasting, or more obvious disease symptoms and mortality. However, adult fish with significant parasite loads also exhibit wasting and reduced reproductive capacity.

\section{Life Cycles}

Nematode life cycles differ depending upon the species of nematode. Typical life cycles are summarized in Figures 6-8. Organisms that contain the reproductive adult stages of nematodes are known as final or definitive hosts. Organisms that are required for the completion of a nematode life cycle but do not contain the final reproductive adult stage are known as intermediate hosts.

There are two major categories of nematode life cycles - DIRECT and INDIRECT.

If the nematode has a DIRECT life cycle (Figure 6), then it does not need an intermediate host and infection can spread directly from one fish to another by ingestion of eggs or larvae.

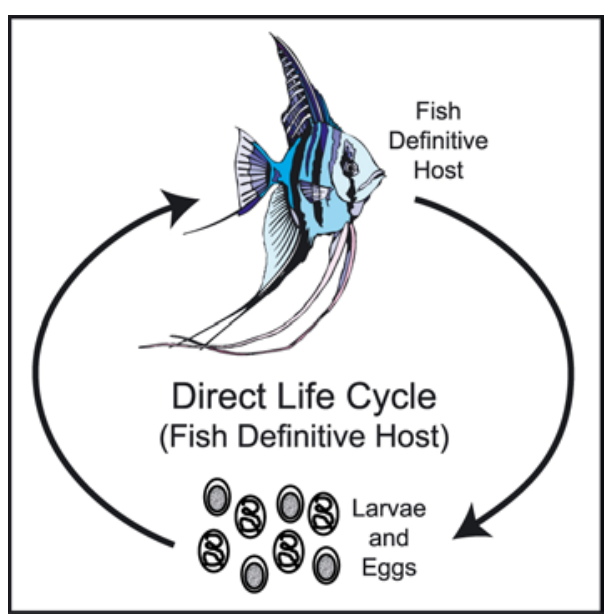

Figure 6. Direct life cycle. Nematode can infect fish directly without the need for an intermediate host.
If the nematode has an INDIRECT life cycle (Figures 7 and 8), the eggs or larvae are excreted into the water and, during development, immature stages pass through at least two different types of organisms, one of which may be a fish. Depending upon the role of the fish, indirect life cycles can also be subcategorized by whether the fish is (i) the definitive (final) host or (ii) an intermediate host.

i) Indirect life cycle where the fish is the final (definitive) host (Figure 7) If the fish is the definitive or final host, then the nematode will enter another organism - usually an aquatic invertebrate such as a copepod, side swimmer (such as Gammarus spp.), tubifex worm, or insect larva - in which it will further develop prior to being eaten by a fish. After being eaten by the correct species of fish, some species of nematode reach sexual maturity and reproduce in these fish. In such a case, the fish are considered the "definitive" or "final" host for these nematode species.

\section{ii) Indirect life cycle where the fish is an}

intermediate host (Figure 8) Nematodes that have this type of life cycle use fish species as intermediate hosts only. The final host (which contains the reproductive adult stage of the nematode) may be a piscivorous (fish-eating) fish, bird or mammal.

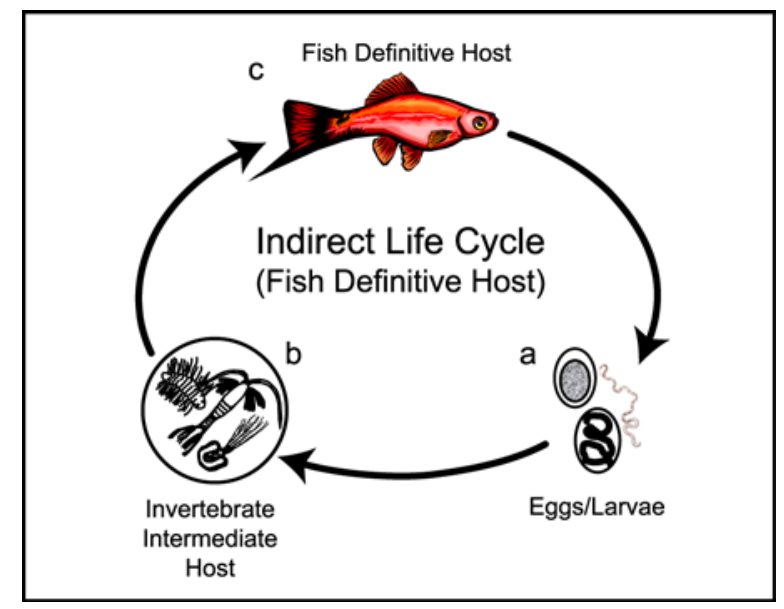

Figure 7. Indirect life cycle where the fish is the final (definitive) host. The nematode eggs/larvae (a) enter an aquatic invertebrate intermediate host (b), such as a copepod, tubifex worm, or insect larva, prior to being eaten by or entering the final host fish (c). 


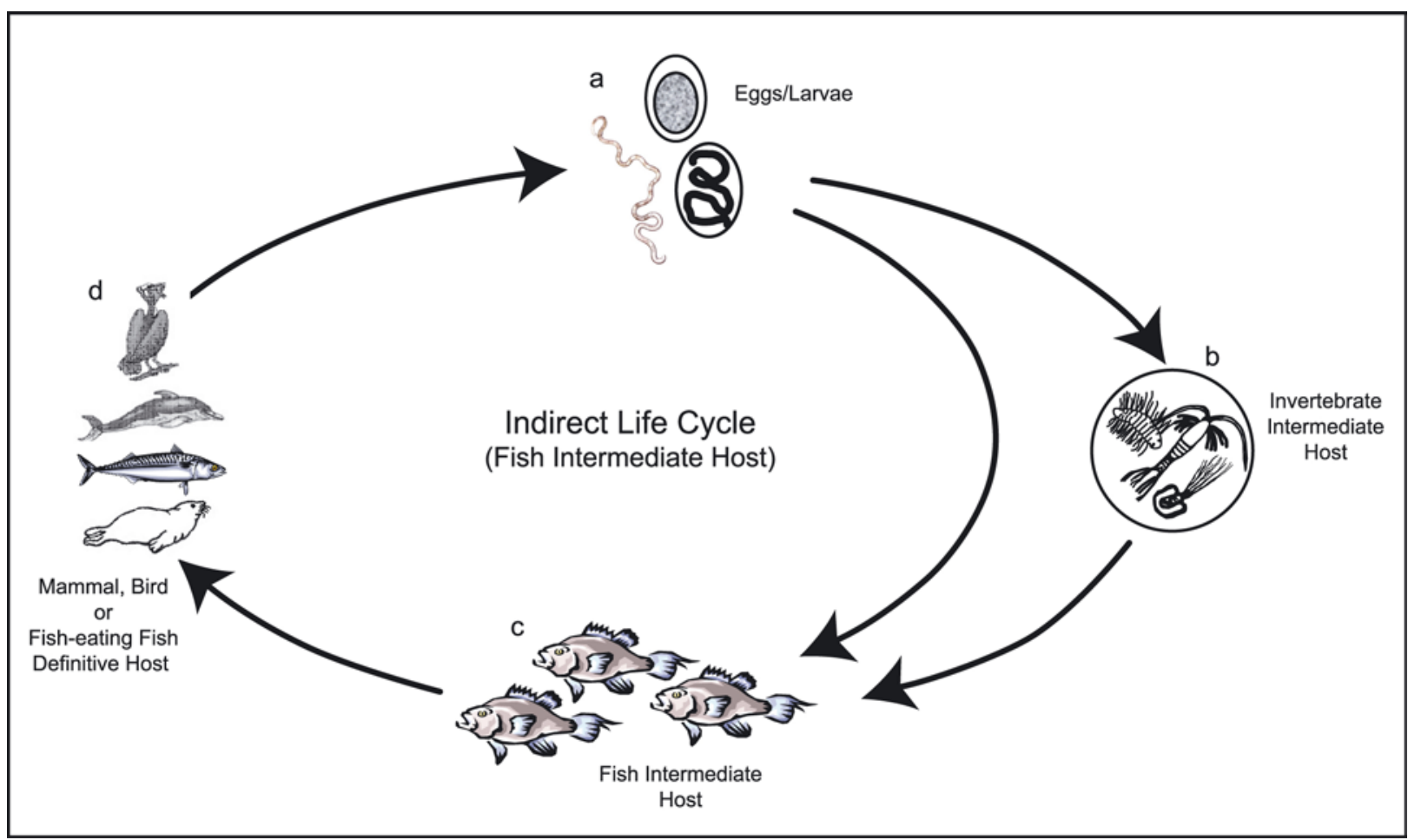

Figure 8. Indirect life cycle where the fish is an intermediate host. The nematode eggs/larvae (a) enter an invertebrate intermediate host (b) OR a fish intermediate host (c) prior to being eaten by or entering the final host, a fish-eating mammal, bird, or fish (d).

Some nematodes have the ability to survive in "alternative" organisms, known as "paratenic" hosts. These hosts are not required for completion of the life cycle but they can contain infective nematode life stages and be a source of infection (Figure 9). They can be fish, worms or other aquatic organisms that eat the nematode eggs or larvae.

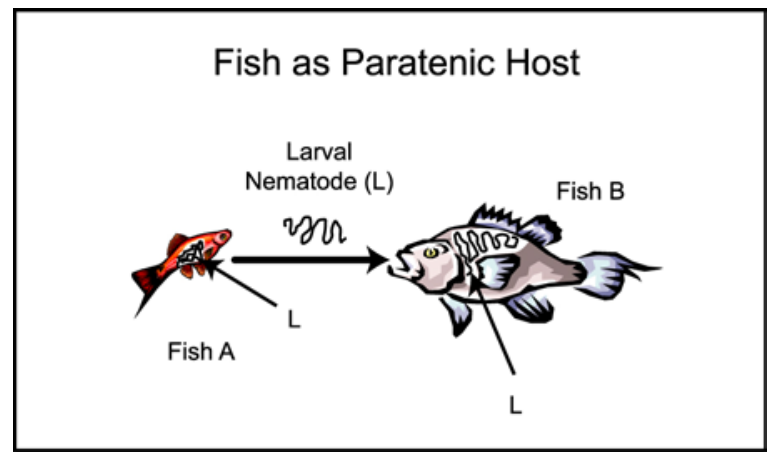

Figure 9. Paratenic host. Fish A contains a larval nematode $(L)$. When Fish $A$ is eaten by Fish $B$, larval nematode $(L)$ migrates out of Fish A into Fish B's body. Fish $B$ can be considered a paratenic host because it is not required for completion of the nematode life cycle, but can still serve as a host.

\section{General Notes \\ Diagnosis}

Intestinal nematode infections should be suspected if fish eat well but are thin ("wasted"), if brood stock production declines over time, or if juveniles grow more slowly than expected or are stunted (unusually small). Infections by nematodes in the skin or muscle may be visible or be suspected if lumps or grub-like growths are seen. As described in the section 'Disease in Fish', fish infected with Eustrongylides species often have grossly distended abdomens, but appear and behave as normal. Nematodes may also infect other tissues and organs in the body resulting in symptoms related to the organ system affected and the degree of damage.

Since other diseases can create the conditions described above, positive identification of infection by nematodes is important. This identification can be made by complete necropsy of a representative sample of the affected population or by biopsy of external lesions. 
An alternative to necropsy for identification of intestinal infections is collection of fresh fecal samples followed by microscopic evaluation for presence of nematode eggs, larvae, or adults.

Feces to be examined must be absolutely fresh, since feces that have been in a tank for even just a few minutes may contain free-living nematodes that are not parasitic and are not a concern for fish health. These free-living nematodes are often difficult to distinguish from parasitic nematodes. If possible, multiple fecal samples from the same group of fish should be taken over time. This increases the likelihood of identifying early nematode life stages.

Even though periodic checks of brood stock and juveniles can be made by examining fresh fecal matter for the presence of nematode eggs or larvae, the sacrifice and necropsy of a small representative group of fish is the most informative method. For pets or valuable individuals examination of fecal material may be the best option for diagnosis of infection.

\section{Treatment}

Prior to administration of deworming medications (anthelminthic), the problem should be properly diagnosed by a fish health specialist. After verification of infection, alternative therapies can be discussed. Food fish and game fish producers will have more limited treatment options than those available to ornamental fish producers. There are no Food and Drug Administration (FDA)-approved drugs for use in the treatment of nematode infections in baitfish, ornamental fish, or food fish, but ornamental fish are considered a low regulatory priority by the FDA.

For intestinal nematode infections of ornamental fish, several anthelminthics (dewormers) are available. Two effective and commonly used dewormers are fenbendazole and levamisole. Fenbendazole can only be used as a feed additive at the rate of 1.14 grams per pound of food fed for three days, with a repeat treatment in two to three weeks. Levamisole can be used both in the feed and as a bath treatment. One effective oral dose is 1.8 grams of levamisole per pound of food fed once a week for three weeks. One effective bath treatment dose is $2 \mathrm{ppm}(\mathrm{mg} / \mathrm{liter})$ levamisole for 24 hours, with a repeat treatment in two to three weeks. However, be sure to consult with a fish health specialist before commencing any treatment and always follow their recommendations.
Control of nematodes that infect areas of the fish other than the gastrointestinal tract is more problematic. For example, Eustrongylides species are typically found encysted within the body cavity outside of the intestinal tract, so common dewormers are ineffective. In fact, surgical removal is the only way to rid fish of internal worms that are not found in the intestine. Fish that contain nematode larvae in their muscles or external areas may be treated by surgical removal of the larvae. This approach is not practical aquaculture production, and sites of surgery may be unsightly or become infected. In general, affected fish must be culled.

\section{Prevention}

Prevention is always the best option, especially against those species of nematode that infect areas other than the gastrointestinal tracts of fish. Ponds that have not been cleaned or sterilized prior to restocking are at greater risk of harboring numbers of intermediate hosts (e.g., tubifex worms). Cleaning and sterilizing ponds is an effective way of reducing the numbers of the intermediate hosts of some nematode species. Preventive medicine can also help the producer avoid illness and production declines.

Preventive medicine includes periodic checks of susceptible juveniles or brood stock (by regular fecal examinations and necropsies of small numbers of fish) and reducing the presence of intermediate hosts, including fish-eating birds and tubifex worms, as mentioned above.

Producers who use live side swimmers (such as Gammarus spp.), insect larvae, tubifex worms, or copepods as food must also be aware that they may be, inadvertently, introducing nematode larvae into their stocks. So, even if these producers obtain their live food from a source that guarantees it is not grown in water containing fish, accessible by fisheating birds or contains other hosts (all sources of possible infection), it is still important for these producers to practice preventative techniques, such as fecal checks, pond sterilization and necropsies on a regular basis. 


\section{Representative Nematode (Roundworm) Species}

\section{Capillaria Species}

The Capillarids as a group can infect a wide variety of fish hosts. Capillaria pterophylli is a relatively common nematode seen in the intestines of cichlids (including angelfish and discus). Capillaria species are also seen in cyprinids, gouramis, tetras and other species of fish.

Capillaria females are easily identified when they are carrying the brown, barrel-shaped eggs with a plug-like structure on each end (Figure 10).

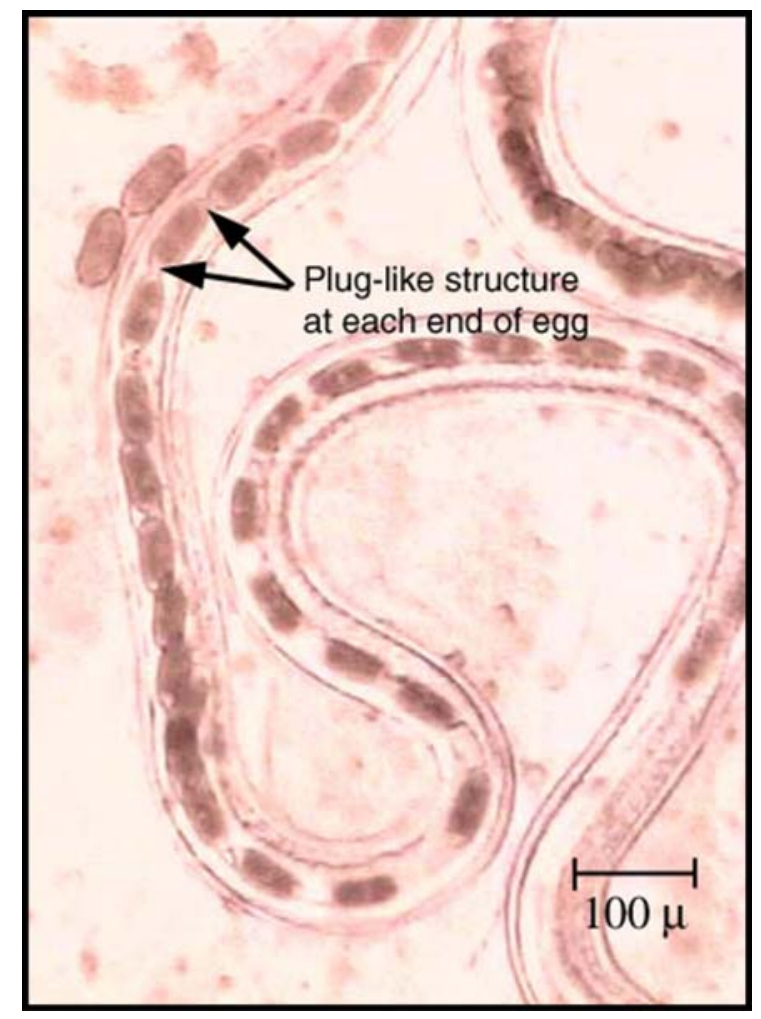

Figure 10. Capillaria female showing eggs with plug-like structures at each end.

Capillaria species have direct life cycles, and can spread from one fish to another by ingestion of infective eggs. It may take Capillaria pterophylli eggs up to three weeks at $68-73^{\circ} \mathrm{F}$ (less time at warmer temperatures) before they contain embryos developed enough to be infective when ingested by a fish. The length of time required from infection until the mature adult parasites are producing eggs or larvae is approximately three months at these temperatures. Even though Capillaria species have direct life cycles, a tubifex worm may act as a paratenic (alternative) host and "carry" infective stages of Capillaria to the fish that consumes them.

\section{Diagnosis}

Capillaria species are relatively transparent and, if immature or male nematodes only are present, may be easily missed during necropsy. However, Capillarid infection is relatively easy to diagnose when females containing the typical barrel-shaped eggs with a polar plug on each end (opercula) are present in the intestinal tract or the eggs alone are present.

\section{Treatment}

Capillarid infections can be treated with dewormers such as levamisole or fenbendazole (see General Treatment Notes). To avoid reinfection, organic debris and feces should be removed as often as possible, especially following deworming treatment.

\section{Prevention}

Brood stock should be evaluated for the presence of nematodes prior to use and periodically throughout the breeding period. Proper sanitation will help prevent the spread of nematodes and reduce infection loads. Live foods, such as oligochaete worms (e.g., tubifex worms), may act as carriers, and their use should be avoided if possible.

\section{Eustrongylides Species}

Eustrongylides species can be found within the body cavity, encapsulated on the liver and other organs, but outside the intestinal tract of fish. Eustrongylid nematodes can affect a number of different species, including yellow perch, pumpkin seed, mummichug, guppies, gar, danios, and angelfish. Affected fish typically have bloated abdomens (dropsy), as the nematodes frequently migrate into the body cavity and can be quite large.

Eustrongylids are typically very long, coiled, and red (due to presence of hemoglobin), and an infected fish often has more than one nematode in its body cavity (Figure 11). If feeder fish containing Eustrongylides species are fed to other fish, the nematodes can migrate out of the feeder fish and into the muscles or other organs of the fish that just consumed them. After migrating into the muscle, this nematode can cause lesions that look superficially similar to a grub. 


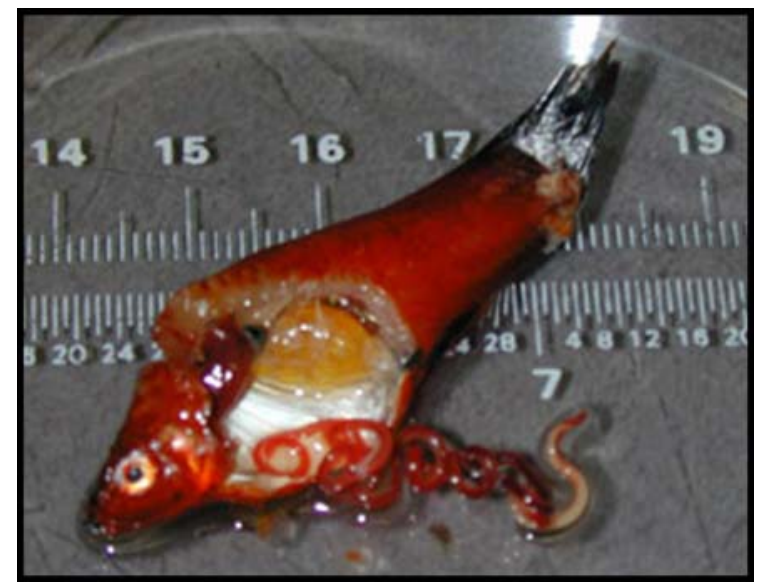

Figure 11. Several Eustrongylides nematodes, exhibiting typical coiling (and red coloration when viewed on the EDIS web site or printed in color), from the body cavity of a fish. (Ruler shows measurements in inches on the top and millimeters on the bottom.)

Eustrongylides species have complex, indirect life cycles. Adult Eustrongylides tubifex and other Eustrongylid nematodes are found in fish-eating birds. The eggs are shed by the birds into ponds, where they develop into a life stage that is consumed by an oligochaete worm, such as the tubifex worm. Within these tubifex worms, the nematodes develop still further into a third larval stage, known as an "L3", which is the life stage of the Eustrongylides group that can infect fish when eaten. Once the tubifex worm containing the L3 stages is eaten by a fish and digested, the nematodes migrate (within the fish) into the body cavity and, frequently, over the external surface of internal organs such as the liver. Some recent studies, however, suggest that Eustrongylides ignotus, commonly found in mosquitofish and which is a close relative of Eustrongylides tubifex, may be able to complete its life cycle without the need for a tubifex worm (D. Coyner, University of Florida, unpublished data; D. Forrester, University of Florida, personal comm.).

The eggs of all Eustrongylides species are very tough and can easily survive for some time in fish ponds. At about $77^{\circ} \mathrm{F}$, it can take anywhere from three months to four and one-half months from the time the bird infects the pond with Eustrongylides eggs until the time fish become infected. This means that, after sterilization of ponds, if fisheating birds do infect the ponds with Eustrongylides' eggs, the producer may not see a problem until harvesting the fish, 3-4 months later, as this is approximately the time required for the eggs to hatch and become the L3 stage which infects the fish. After this 3-4 month period, fish raised in ponds with a population of fish-eating birds have an even greater chance of becoming infected as the number of nematodes increase over time.

\section{Diagnosis}

As Eustrongylides species infect areas outside the gastrointestinal tracts of fish, necropsy of a small group of affected fish is the only sure method to identify them. During necropsy Eustrongylides species, such as E. tubifex, are easily identified from their location in the fish (encysted in mesenteries of the body cavity or in muscle), their red coloration, and their relatively long bodies (11-83 $\mathrm{mm})$; however, there are other nematodes that may appear similar, so positive identification will require examination by an expert.

\section{Treatment}

Other than mechanical removal, there is no effective treatment for these nematodes due to the location of the larvae within the coelomic cavity or in the muscle. Culling affected fish is recommended.

\section{Prevention}

Removal of the final host (fish-eating birds) or any intermediate hosts (tubificid worms or other oligochaetes) will help reduce the infection rate. Sanitation of ponds or tanks will help remove any intermediate hosts. Sanitation of ponds at the very minimum of once or twice a year is recommended.

\section{Camallanus Species}

Camallanus species (Figure 12) infect the gastrointestinal tract of cichlids, live-bearers (including guppies and swordtails), and other species of freshwater fish. Usually, the first evidence of infection is a red, worm-like animal protruding from the anus of a fish.

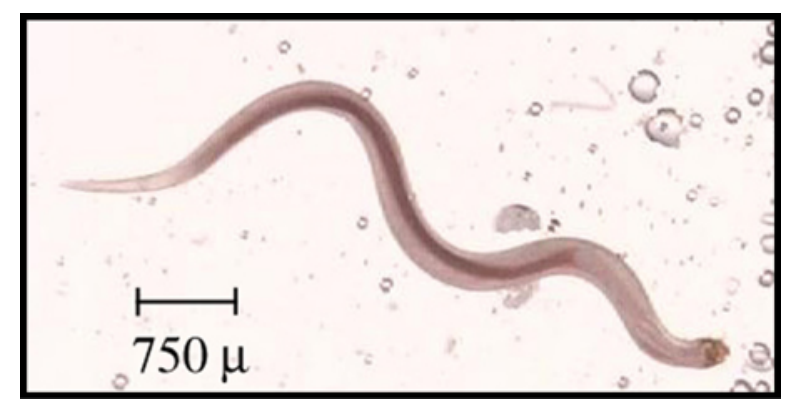

Figure 12. Juvenile Camallanus nematode from a fire mouth cichlid. 
Camallanus species have indirect life cycles and are live-bearing nematodes. They are considered "ovoviviparous" ("ovo" is the scientific term for "egg" and "viviparous" means live-bearing), as females incubate the eggs which hatch into larvae within their bodies (Figure 13). These larvae are excreted into the water with the fish's feces and are ingested by a copepod or other crustacean. Within the copepod, the larvae develop further into a thirdstage larvae. After the copepod, containing the third-stage larvae, is eaten by the appropriate fish host, the larvae migrate out into the fish, develop into reproductive adults, and the life cycle is complete.

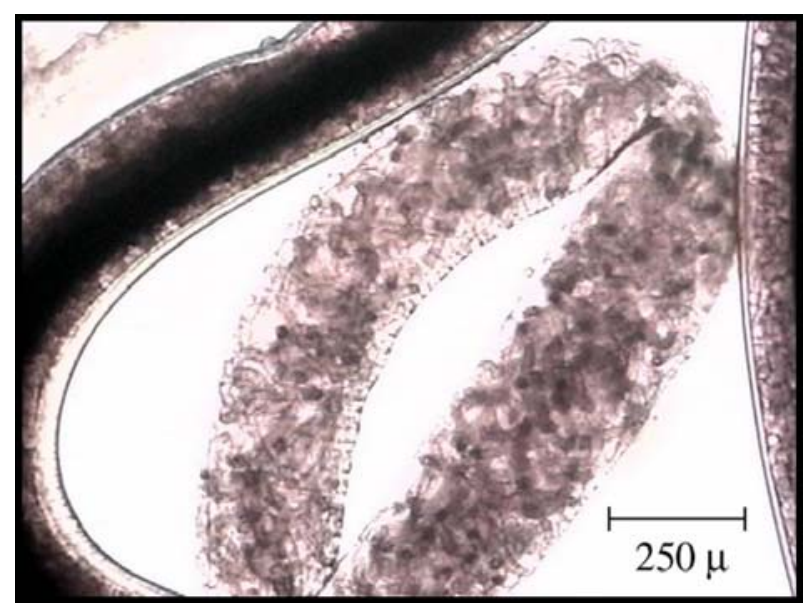

Figure 13. Close up of a Camallanus sp. female showing larvae inside.

\section{Diagnosis}

Camallanus species can be identified by their red color; their location further toward the posterior of the intestinal tract than other worm-like parasites (typically very near, and often protruding from, the anus of the fish); the presence of a buccal capsule (mouth structure) that is divided into two lateral valves, giving the mouth a slit like appearance; and, if gravid females are present, the presence of both eggs and larvae within their bodies. Positive identification will require the assistance of an expert.

\section{Treatment}

Because Camallanus nematodes are located within the intestinal tract, common dewormers should be effective.

\section{Prevention}

As a copepod or other crustacean host is required to complete the life cycle, avoiding the use of copepods or related crustaceans as food items will help reduce potential for infection. Evaluation of brood stock for the presence of parasites prior to use will also aid in identifying carriers.

\section{Contracaecum Species}

Larval stages of the Contracaecum species that infect freshwater fish are usually found as adults in fish-eating birds, such as cormorants and pelicans. Larval stages (Figure 14) are seen in cyprinids (carp and related species), ictalurids (channel catfish), centrarchids (sunfish and bass), tilapia and other cichlids, and percids (perch). On the other hand, the larval stage of the Contracaecum species that infect marine fish, such as whiting, capelin, and cod, are typically found as adults in seals.

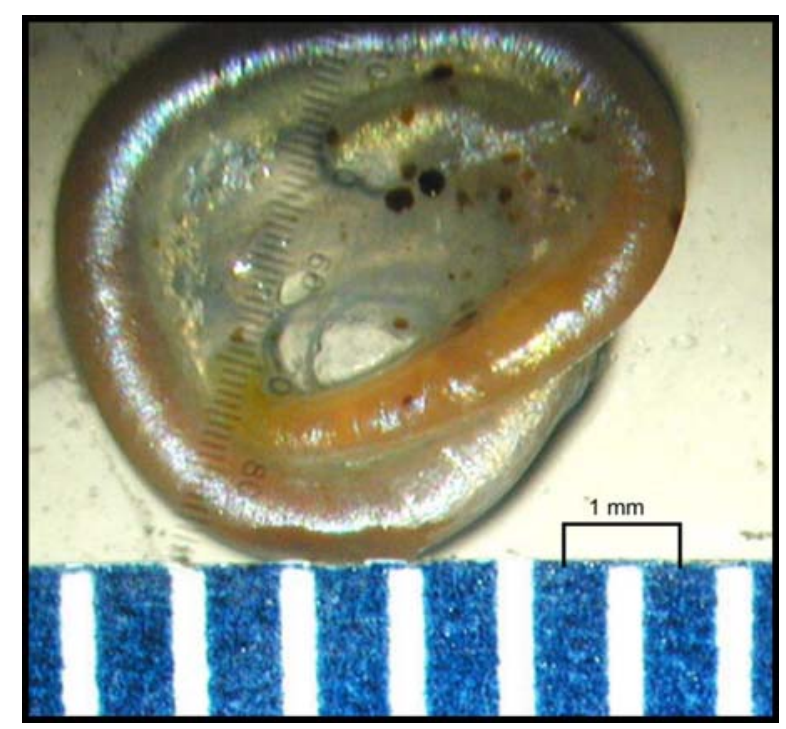

Figure 14. Larval Contracaecum sp. from a freshwater fish, showing typical coiled position.

As implied above, Contracaecum species have complex life cycles. The eggs are released by gravid females into the intestinal tracts of their definitive (final) hosts, where they are excreted into the water with the feces. The eggs hatch and the free-living larvae develop into the infective thirdstage larvae (L3). These L3 larvae are then ingested by an invertebrate intermediate host, within which they develop even further. This invertebrate host is then ingested by the fish intermediate host, where it remains until the intermediate fish host is eaten by the final host (a fish-eating bird or mammal). Some species of Contracaecum (e.g., C. spiculigerum) appear to have no specificity for fish hosts, so they are able to use a wide variety of fish species as intermediate hosts. 
Contracaecum species have been found in several locations within the body cavity of fish, including the liver, muscles, heart, and swim bladder.

\section{Diagnosis}

During necropsy of a small group of fish, tentative identification of infection by Contracaecum species can be made based upon finding larval nematodes in the locations listed above. However, because other nematodes have larvae which may look similar to larvae of Contracaecum, positive identification of Contracaecum infection needs to be made by an expert.

\section{Treatment}

Because of the location of larval nematodes in the freshwater fish intermediate host, chemical treatments, such as dewormers and chemical baths, are not an option and may even incite an immune reaction in the fish, ultimately doing more harm than good.

\section{Prevention}

Elimination of final hosts (bird or mammal) and invertebrate intermediate hosts from the aquaculture site will help reduce and, possibly, eliminate infection.

\section{Summary}

Nematodes, or roundworms, have been found in numerous species of fish. Because they are similar to other worm-like parasites of fish, proper diagnosis is important for effective treatment. Working with a fish health specialist will insure proper diagnosis and legal treatment or prevention. Producers of food and game fish have more limited options for the treatment of infected fish than will the producers of ornamental fish because ornamental fish are considered a low regulatory priority by the FDA.

Small numbers of nematodes may be present in fish without causing significant symptoms. In more severely infected fish, evidence of disease can inlcude emaciation (wasting or significant loss of body mass), nodules or masses present in skin or muscle, stunted growth, abnormal swimming, lethargy, or death.

Depending upon the species of fish and the species of nematode, fish may be final hosts (containing adult nematodes) or intermediate hosts (containing larval nematodes). Some species of nematodes that infect fish can be transmitted directly from one fish in the population to another (direct life cycle). Other species of nematodes require additional hosts to complete their life cycle, commonly an invertebrate host or a fish, bird, or mammal as final host. Consequently, correct identification of the nematode is critical for proper management of the disease.

Nematodes found within the gastrointestinal tracts of ornamental fish can be treated with appropriate dewormers. Nematodes found in other areas of ornamental fish cannot be removed through chemical means, and the fish in such cases must be culled. Control of intermediate or final hosts will help break the life cycles of these parasites. In addition, proper sanitation of ponds or tanks will help reduce the abundance of infective stages.

\section{References}

Dick, T.A. and A. Choudhury. 1995. Phylum Nematoda. In Fish Diseases and Disorders, pp. 415-446, Chapter 11, Volume 1, Protozoan and Metazoan Infections, P.T.K. Woo (editor), CAB International, Cambridge, UK.

Carpenter, J.W., Mashima, T.Y. and Rupiper, D.J. 1996. Exotic Animal Formulary. First Ed. Greystone Publications. Manhattan, KS. 310 pp.

Hoffman, G.L. 1999. Parasites of North American Freshwater Fishes. Second Edition. Comstock Publishing Associates, Ithaca, NY.

Measures, L.N. 1987. Epizootiology, pathology, and description of Eustrongylides tubifex (Nematoda: Dioctophymatoidea) in fish. Canadian Journal of Zoology 66: 2212-2222.

Measures, LN. 1988. The Development of Eustrongylides tubifex (Nematoda: Dioctophymatoidea) in Oligochaetes. Journal of Parasitology 74(2): 294-304.

Noga, E.J. 1996. Fish Disease: Diagnosis and Treatment. Mosby-Year Book, Inc., St. Louis, MO, pp. 166-170. 